\title{
On the Global Character of the System of Piecewise Linear Difference Equations $x_{n+1}=\left|x_{n}\right|-y_{n}-1$ and $y_{n+1}=x_{n}-\left|y_{n}\right|$
}

\author{
Wirot Tikjha, ${ }^{1,2}$ Yongwimon Lenbury, ${ }^{1,2}$ \\ and Evelina Giusti Lapierre ${ }^{3}$ \\ ${ }^{1}$ Department of Mathematics, Faculty of Science, Mahidol University, Rama 6 Road Bangkok, \\ 10400, Thailand \\ ${ }^{2}$ Center of Excellence in Mathematics, PERDO Commission on Higher Education, Si Ayudhya Road, \\ Bangkok 10400, Thailand \\ ${ }^{3}$ John Hazen White School of Arts and Sciences, Department of Mathematics, \\ Johnson and Wales University, 8 Abbott Park Place, Providence, RI 02903, USA
}

Correspondence should be addressed to Yongwimon Lenbury, scylb@mahidol.ac.th

Received 23 June 2010; Revised 4 September 2010; Accepted 2 December 2010

Academic Editor: Donal O’Regan

Copyright (C) 2010 Wirot Tikjha et al. This is an open access article distributed under the Creative Commons Attribution License, which permits unrestricted use, distribution, and reproduction in any medium, provided the original work is properly cited.

We consider the system in the title where the initial condition $\left(x_{0}, y_{0}\right) \in \mathbf{R}^{2}$. We show that the system has exactly two prime period-5 solutions and a unique equilibrium point $(0,-1)$. We also show that every solution of the system is eventually one of the two prime period- 5 solutions or else the unique equilibrium point.

\section{Introduction}

In this paper, we consider the system of piecewise linear difference equations

$$
\begin{gathered}
x_{n+1}=\left|x_{n}\right|-y_{n}-1, \\
y_{n+1}=x_{n}-\left|y_{n}\right|,
\end{gathered}
$$

where the initial condition $\left(x_{0}, y_{0}\right) \in \mathbf{R}^{2}$. We show that every solution of System (1.1) is eventually either one of two prime period- 5 solutions or else the unique equilibrium point $(0,-1)$. 
System (1.1) was motivated by Devaney's Gingerbread man map [1, 2]

$$
x_{n+1}=\left|x_{n}\right|-x_{n-1}+1
$$

or its equivalent system of piecewise linear difference equations $[3,4]$

$$
\begin{gathered}
x_{n+1}=\left|x_{n}\right|-y_{n}+1, \\
y_{n+1}=x_{n},
\end{gathered} \quad n=0,1,2, \ldots .
$$

We believe that the methods and techniques used in this paper will be useful in discovering the global character of solutions of similar systems, including the Gingerbread man map.

\section{The Global Behavior of the Solutions of System (1.1)}

System (1.1) has the equilibrium point $(\bar{x}, \bar{y}) \in \mathbf{R}^{2}$ given by

$$
(\bar{x}, \bar{y})=(0,-1)
$$

System (1.1) has two prime period-5 solutions,

$$
\begin{gathered}
P_{5}^{1}=\left(\begin{array}{ll}
x_{0}=0, & y_{0}=1 \\
x_{1}=-2, & y_{1}=-1 \\
x_{2}=2, & y_{2}=-3 \\
x_{3}=4, & y_{3}=-1 \\
x_{4}=4, & y_{4}=3
\end{array}\right), \\
P_{5}^{2}=\left(\begin{array}{cc}
x_{0}=0, & y_{0}=\frac{1}{7} \\
x_{1}=-\frac{8}{7}, & y_{1}=-\frac{1}{7} \\
x_{2}=\frac{2}{7}, & y_{2}=-\frac{9}{7} \\
x_{3}=\frac{4}{7}, & y_{3}=-1 \\
x_{4}=\frac{4}{7}, & y_{4}=-\frac{3}{7} .
\end{array}\right) .
\end{gathered}
$$


Set

$$
\begin{aligned}
& l_{1}=\{(x, y): x \geq 0, y=0\}, \\
& l_{2}=\{(x, y): x=0, y \geq 0\}, \\
& l_{3}=\{(x, y): x<0, y=0\}, \\
& l_{4}=\{(x, y): x=0, y<0\}, \\
& Q_{1}=\{(x, y): x>0, y>0\}, \\
& Q_{2}=\{(x, y): x<0, y>0\}, \\
& Q_{3}=\{(x, y): x<0, y<0\}, \\
& Q_{4}=\{(x, y): x>0, y<0\} .
\end{aligned}
$$

Theorem 2.1. Let $\left(x_{0}, y_{0}\right) \in \mathbf{R}^{2}$. Then there exists an integer $\mathcal{N} \geq 0$ such that the solution $\left\{\left(x_{n}, y_{n}\right)\right\}_{n=\mathcal{N}}^{\infty}$ is eventually either the prime period-5 solution $P_{5}^{1}$, the prime period-5 solution $P_{5}^{2}$, or else the unique equilibrium point $(0,-1)$.

The proof is a direct consequence of the following lemmas.

Lemma 2.2. Suppose there exists an integer $M \geq 0$ such that $-1 \leq x_{M} \leq 0$ and $y_{M}=-x_{M}-1$. Then $\left(x_{M+1}, y_{M+1}\right)=(0,-1)$, and so $\left\{\left(x_{n}, y_{n}\right)\right\}_{n=M+1}^{\infty}$ is the equilibrium solution.

Proof. Note that

$$
\begin{gathered}
x_{M+1}=\left|x_{M}\right|-y_{M}-1=-x_{M}-\left(-x_{M}-1\right)-1=0, \\
y_{M+1}=x_{M}-\left|y_{M}\right|=x_{M}-\left(x_{M}+1\right)=-1,
\end{gathered}
$$

and so the proof is complete.

Lemma 2.3. Suppose there exists an integer $M \geq 0$ such that $x_{M} \geq 1$ and $y_{M}=x_{M}-1$. Then $\left(x_{M+1}, y_{M+1}\right)=(0,1)$, and so $\left\{\left(x_{n}, y_{n}\right)\right\}_{n=M+1}^{\infty}$ is $P_{5}^{1}$.

Proof. We have

$$
\begin{gathered}
x_{M+1}=\left|x_{M}\right|-y_{M}-1=x_{M}-\left(x_{M}-1\right)-1=0, \\
y_{M+1}=x_{M}-\left|y_{M}\right|=x_{M}-\left(x_{M}-1\right)=1,
\end{gathered}
$$

and so the proof is complete.

Lemma 2.4. Suppose there exists an integer $M \geq 0$ such that $x_{M}=0$ and $y_{M} \geq 0$. Then the following statements are true.

(1) $x_{M+5}=0$.

(2) If $y_{M}>1 / 4$, then $\left\{\left(x_{n}, y_{n}\right)\right\}_{n=M+5}^{\infty}$ is $P_{5}^{1}$.

(3) If $0 \leq y_{M} \leq 1 / 4$, then $y_{M+5}=8 y_{M}-1$. 
Proof. We have $x_{M}=0$ and $y_{M} \geq 0$. Then

$$
\begin{gathered}
x_{M+1}=\left|x_{M}\right|-y_{M}-1=-y_{M}-1<0, \\
y_{M+1}=x_{M}-\left|y_{M}\right|=-y_{M} \leq 0, \\
x_{M+2}=\left|x_{M+1}\right|-y_{M+1}-1=2 y_{M} \geq 0, \\
y_{M+2}=x_{M+1}-\left|y_{M+1}\right|=-2 y_{M}-1<0, \\
x_{M+3}=\left|x_{M+2}\right|-y_{M+2}-1=4 y_{M} \geq 0, \\
y_{M+3}=x_{M+2}-\left|y_{M+2}\right|=-1, \\
x_{M+4}=\left|x_{M+3}\right|-y_{M+3}-1=4 y_{M} \geq 0, \\
y_{M+4}=x_{M+3}-\left|y_{M+3}\right|=4 y_{M}-1, \\
x_{M+5}=\left|x_{M+4}\right|-y_{M+4}-1=0,
\end{gathered}
$$

and so statement (1) is true.

If $y_{M}>1 / 4$, then $y_{M+5}=x_{M+4}-\left|y_{M+4}\right|=1$. That is, $\left(x_{M+5}, y_{M+5}\right)=(0,1)$ and so statement (2) is true.

If $0 \leq y_{M} \leq 1 / 4$, then $y_{M+5}=x_{M+4}-\left|y_{M+4}\right|=8 y_{M}-1$, and so statement (3) is true.

Lemma 2.5. Suppose there exists an integer $M \geq 0$ such that $x_{M}=0$ and $y_{M}<-1$. Then the following statements are true.

(1) $x_{M+4}=0$.

(2) If $-3 / 2<y_{M}<-1$, then $y_{M+4}=-4 y_{M}-5$.

(3) If $y_{M} \leq-3 / 2$, then $\left\{\left(x_{n}, y_{n}\right)\right\}_{n=M+4}^{\infty}$ is $P_{5}^{1}$.

Proof. We have $x_{M}=0$ and $y_{M}<-1$. Then

$$
\begin{gathered}
x_{M+1}=\left|x_{M}\right|-y_{M}-1=-y_{M}-1>0, \\
y_{M+1}=x_{M}-\left|y_{M}\right|=y_{M}<0, \\
x_{M+2}=\left|x_{M+1}\right|-y_{M+1}-1=-2 y_{M}-2>0, \\
y_{M+2}=x_{M+1}-\left|y_{M+1}\right|=-1, \\
x_{M+3}=\left|x_{M+2}\right|-y_{M+2}-1=-2 y_{M}-2>0, \\
y_{M+3}=x_{M+2}-\left|y_{M+2}\right|=-2 y_{M}-3, \\
x_{M+4}=\left|x_{M+3}\right|-y_{M+3}-1=0,
\end{gathered}
$$

and so statement $(1)$ is true.

Now if $-3 / 2<y_{M}<-1$, then $y_{M+3}=-2 y_{M}-3<0$. Thus $y_{M+4}=x_{M+3}-\left|y_{M+3}\right|=$ $-4 y_{M}-5$, and so statement (2) is true.

Lastly, if $y_{M} \leq-3 / 2$, then $y_{M+3}=-2 y_{M}-3 \geq 0$. Thus $y_{M+4}=x_{M+3}-\left|y_{M+3}\right|=1$; that is, $\left(x_{M+4}, y_{M+4}\right)=(0,1)$ and so statement (3) is true. 
Lemma 2.6. Suppose there exists an integer $M \geq 0$ such that $x_{M} \geq 0$ and $y_{M}=0$. Then the following statements are true.

(1) If $x_{M} \geq 1$, then $\left\{\left(x_{n}, y_{n}\right)\right\}_{n=M+2}^{\infty}$ is $P_{5}^{1}$.

(2) If $1 / 4<x_{M}<1$, then $\left\{\left(x_{n}, y_{n}\right)\right\}_{n=M+6}^{\infty}$ is $P_{5}^{1}$.

(3) If $0 \leq x_{M} \leq 1 / 4$, then $x_{M+6}=0$ and $y_{M+6}=8 x_{M}-1$.

Proof. First consider the case $x_{M} \geq 1$ and $y_{M}=0$. Then

$$
\begin{gathered}
x_{M+1}=\left|x_{M}\right|-y_{M}-1=x_{M}-1 \geq 0, \\
y_{M+1}=x_{M}-\left|y_{M}\right|=x_{M}>0, \\
x_{M+2}=\left|x_{M+1}\right|-y_{M+1}-1=-2, \\
y_{M+2}=x_{M+1}-\left|y_{M+1}\right|=-1,
\end{gathered}
$$

and so statement (1) is true.

Next consider the case $0 \leq x_{M}<1$ and $y_{M}=0$. Then

$$
\begin{gathered}
x_{M+1}=\left|x_{M}\right|-y_{M}-1=x_{M}-1<0, \\
y_{M+1}=x_{M}-\left|y_{M}\right|=x_{M} \geq 0, \\
x_{M+2}=\left|x_{M+1}\right|-y_{M+1}-1=-2 x_{M} \leq 0, \\
y_{M+2}=x_{M+1}-\left|y_{M+1}\right|=-1, \\
x_{M+3}=\left|x_{M+2}\right|-y_{M+2}-1=2 x_{M} \geq 0, \\
y_{M+3}=x_{M+2}-\left|y_{M+2}\right|=-2 x_{M}-1<0, \\
x_{M+4}=\left|x_{M+3}\right|-y_{M+3}-1=4 x_{M} \geq 0, \\
y_{M+4}=x_{M+3}-\left|y_{M+3}\right|=-1, \\
x_{M+5}=\left|x_{M+4}\right|-y_{M+4}-1=4 x_{M} \geq 0, \\
y_{M+5}=x_{M+4}-\left|y_{M+4}\right|=4 x_{M}-1, \\
x_{M+6}=\left|x_{M+5}\right|-y_{M+5}-1=0 .
\end{gathered}
$$

If $1 / 4<x_{M}<1$, then $y_{M+5}=4 x_{M}-1>0$ and so $y_{M+6}=x_{M+5}-\left|y_{M+5}\right|=1$. That is, $\left(x_{M+6}, y_{M+6}\right)=(0,1)$ and so statement (2) is true.

If $0 \leq x_{M} \leq 1 / 4$, then $y_{M+5}=4 x_{M}-1 \leq 0$. Thus $y_{M+6}=x_{M+5}-\left|y_{M+5}\right|=8 x_{M}-1$, and so statement (3) is true.

Lemma 2.7. Suppose there exists an integer $M \geq 0$ such that $x_{M}<-1$ and $y_{M}=0$. Then the following statements are true.

(1) $x_{M+4}=0$.

(2) If $-3 / 2 \leq x_{M}<-1$, then $y_{M+4}=-4 x_{M}-5$.

(3) If $x_{M}<-3 / 2$, then $\left\{\left(x_{n}, y_{n}\right)\right\}_{n=M+4}^{\infty}$ is $P_{5}^{1}$. 
Proof. Let $x_{M}<-1$ and $y_{M}=0$. Then

$$
\begin{gathered}
x_{M+1}=\left|x_{M}\right|-y_{M}-1=-x_{M}-1>0, \\
y_{M+1}=x_{M}-\left|y_{M}\right|=x_{M}<0, \\
x_{M+2}=\left|x_{M+1}\right|-y_{M+1}-1=-2 x_{M}-2>0, \\
y_{M+2}=x_{M+1}-\left|y_{M+1}\right|=-1, \\
x_{M+3}=\left|x_{M+2}\right|-y_{M+2}-1=-2 x_{M}-2>0, \\
y_{M+3}=x_{M+2}-\left|y_{M+2}\right|=-2 x_{M}-3, \\
x_{M+4}=\left|x_{M+3}\right|-y_{M+3}-1=0,
\end{gathered}
$$

and so statement (1) is true.

If $-3 / 2 \leq x_{M}<-1$, then $y_{M+3}=-2 x_{M}-3 \leq 0$. Thus $y_{M+4}=x_{M+3}-\left|y_{M+3}\right|=-4 x_{M}-5$, and so statement (2) is true.

If $x_{M}<-3 / 2$, then $y_{M+3}=-2 x_{M}-3>0$ and $y_{M+4}=x_{M+3}-\left|y_{M+3}\right|=1$. That is, $\left(x_{M+4}, y_{M+4}\right)=(0,1)$ and so $\left\{\left(x_{n}, y_{n}\right)\right\}_{n=M+4}^{\infty}$ is $P_{5}^{1}$ and the proof is complete.

We now give the proof of Theorem 2.1 when $\left(x_{M}, y_{M}\right)$ is in $l_{2}=\{(x, y): x=0, y \geq 0\}$.

Lemma 2.8. Suppose there exists an integer $M \geq 0$ such that $\left(x_{M}, y_{M}\right) \in l_{2}$. Then the following statements are true.

(1) If $0 \leq y_{M}<1 / 7$, then $\left\{\left(x_{n}, y_{n}\right)\right\}_{n=M}^{\infty}$ is eventually the equilibrium solution.

(2) If $y_{M}=1 / 7$, then the solution $\left\{\left(x_{n}, y_{n}\right)\right\}_{n=M+2}^{\infty}$ is $P_{5}^{2}$.

(3) If $y_{M}>1 / 7$, then the solution $\left\{\left(x_{n}, y_{n}\right)\right\}_{n=M}^{\infty}$ is eventually $P_{5}^{1}$.

Proof. (1) We will first show that statement (1) is true. Suppose $0 \leq y_{M}<1 / 7$; for each $n \geq 0$, let

$$
a_{n}=\frac{2^{3 n}-1}{7 \cdot 2^{3 n}}
$$

Observe that

$$
0=a_{0}<a_{1}<a_{2}<\cdots<\frac{1}{7}, \quad \lim _{n \rightarrow \infty} a_{n}=\frac{1}{7}
$$

Thus there exists a unique integer $K \geq 0$ such that $y_{M} \in\left[a_{K}, a_{K+1}\right)$.

We first consider the case $K=0$; that is, $y_{M} \in[0,1 / 8)$. By statements (1) and (3) of Lemma 2.4, $x_{M+5}=0$ and $y_{M+5}=8 y_{M}-1$. Clearly $y_{M+5}<0$, and so

$$
\begin{gathered}
x_{M+6}=\left|x_{M+5}\right|-y_{M+5}-1=-8 y_{M} \leq 0, \\
y_{M+6}=x_{M+5}-\left|y_{M+5}\right|=8 y_{M}-1 .
\end{gathered}
$$


Now $-1<x_{M+6} \leq 0$ and $y_{M+6}=-x_{M+6}-1$, and so by Lemma 2.2, $\left\{\left(x_{n}, y_{n}\right)\right\}_{n=M+7}^{\infty}$ is the equilibrium solution.

Without loss of generality, we may assume $K \geq 1$.

For each integer $n$ such that $n \geq 0$, let $D(n)$ be the following statement:

$$
\begin{gathered}
x_{M+5 n+5}=0 \\
y_{M+5 n+5}=2^{3(n+1)} y_{M}-\left(\frac{2^{3(n+1)}-1}{7}\right) \geq 0 .
\end{gathered}
$$

Claim 1. $P(n)$ is true for $0 \leq n \leq K-1$.

The proof Claim 1 will be by induction on $n$. We will first show that $p(0)$ is true.

Recall that $x_{M}=0$ and $y_{M} \in\left[a_{K}, a_{K+1}\right) \subset[1 / 8,1 / 7)$. Then by statements (1) and (3) of Lemma 2.4, we have $x_{M+5(0)+5}=0$ and $y_{M+5(0)+5}=8 y_{M}-1$.

Note that,

$$
y_{M+5(0)+5}=8 y_{M}-1=2^{3(0+1)} y_{M}-\left(\frac{2^{3(0+1)}-1}{7}\right) \geq 0
$$

and so $P(0)$ is true. Thus if $K=1$, then we have shown that for $0 \leq n \leq K-1, P(n)$ is true. It remains to consider the case $K \geq 2$. So assume that $K \geq 2$. Let $n$ be an integer such that $0 \leq n \leq K-2$ and suppose $P(n)$ is true. We will show that $D(n+1)$ is true.

Since $p(n)$ is true, we know

$$
x_{M+5 n+5}=0, \quad y_{M+5 n+5}=2^{3(n+1)} y_{M}-\left(\frac{2^{3(n+1)}-1}{7}\right) \geq 0 .
$$

It is easy to verify that for $y_{M} \in[1 / 8,1 / 7)$,

$$
y_{M+5 n+5}=2^{3(n+1)} y_{M}-\left(\frac{2^{3(n+1)}-1}{7}\right)<\frac{1}{4} .
$$

Thus by statements (1) and (3) of Lemma 2.4,

$$
\begin{aligned}
x_{M+5(n+1)+5} & =0 \\
y_{M+5(n+1)+5} & =8\left(y_{M+5 n+5}\right)-1 \\
& =2^{3}\left[2^{3(n+1)} y_{M}-\left(\frac{2^{3(n+1)}-1}{7}\right)\right]-1 \\
& =2^{3 n+6} y_{M}-\frac{2^{3 n+6}}{7}+\frac{2^{3}}{7}-1 \\
& =2^{3(n+2)} y_{M}-\left(\frac{2^{3(n+2)}-1}{7}\right) .
\end{aligned}
$$


Recall that $y_{M} \in\left[a_{K}, a_{K+1}\right)=\left[\left(2^{3 K}-1\right) /\left(7 \cdot 2^{3 K}\right),\left(2^{3(K+1)}-1\right) /\left(7 \cdot 2^{3(K+1)}\right)\right)$.

In particular,

$$
\begin{aligned}
y_{M+5(n+1)+5} & =2^{3(n+2)} y_{M}-\left(\frac{2^{3(n+2)}-1}{7}\right) \\
& \geq 2^{3(n+2)}\left(\frac{2^{3 K}-1}{7 \cdot 2^{3 K}}\right)-\left(\frac{2^{3(n+2)}-1}{7}\right) \\
& =\frac{2^{3 n+3 K+6}}{7 \cdot 2^{3 K}}-\frac{2^{3 n+6}}{7 \cdot 2^{3 K}}-\frac{2^{3 n+6}}{7}+\frac{1}{7} \\
& =\frac{1}{7}\left(1-2^{3[n-(K-2)]}\right) \geq \frac{1}{7}(1-1) \\
& =0
\end{aligned}
$$

and so $P(n+1)$ is true. Thus the proof of the claim is complete. That is, $P(n)$ is true for $0 \leq$ $n \leq K-1$. Specifically, $p(K-1)$ is true, and so

$$
x_{M+5(K-1)+5}=0, \quad y_{M+5(K-1)+5}=2^{3 K} y_{M}-\left(\frac{2^{3 K}-1}{7}\right) \geq 0 .
$$

In particular,

$$
2^{3 K}\left(\frac{2^{3 K}-1}{7 \cdot 2^{3 K}}\right)-\left(\frac{2^{3 K}-1}{7}\right) \leq y_{M+5(K-1)+5}<2^{3 K}\left(\frac{2^{3 K+3}-1}{7 \cdot 2^{3 K+3}}\right)-\left(\frac{2^{3 K}-1}{7}\right) .
$$

That is, $0 \leq y_{M+5(K-1)+5}<1 / 8$, and so by case $K=0,\left\{\left(x_{n}, y_{n}\right)\right\}_{n=M+5 K+7}^{\infty}$ is the equilibrium solution, and the proof of statement (1) is complete.

(2) We will next show that statement (2) is true. Suppose $\left(x_{M}, y_{M}\right)=(0,1 / 7)$. Note that $(0,1 / 7) \in P_{5}^{2}$. Thus the solution $\left\{\left(x_{n}, y_{n}\right)\right\}_{n=M}^{\infty}$ is $P_{5}^{2}$.

(3) Finally, we will show that statement (3) is true. Suppose $y_{M}>1 / 7$. is $P_{5}^{1}$.

First consider $y_{M}>1 / 4$. By statement (2) of Lemma 2.4, the solution $\left\{\left(x_{n}, y_{n}\right)\right\}_{n=M+5}^{\infty}$

Next consider the case $y_{M} \in(1 / 7,1 / 4]$. For each $n \geq 1$, let

$$
b_{n}=\frac{2^{3 n-1}+3}{7 \cdot 2^{3 n-1}}
$$

Observe that

$$
\frac{1}{4}=b_{1}>b_{2}>b_{3}>\cdots>\frac{1}{7}, \quad \lim _{n \rightarrow \infty} b_{n}=\frac{1}{7}
$$

Thus there exists a unique integer $K \geq 1$ such that $y_{M} \in\left(b_{K+1}, b_{K}\right]$. 
Note that the statement $P(n)$ which we stated and proved in the proof of statement (1) of this lemma still holds. Specifically $P(K-1)$ is true, and so

$$
x_{M+5(K-1)+5}=0, \quad y_{M+5(K-1)+5}=2^{3 K} y_{M}-\left(\frac{2^{3 K}-1}{7}\right) \geq 0 .
$$

Recall that for $y_{M} \in\left(b_{K+1}, b_{K}\right]$.

In particular,

$$
y_{M+5 K}=2^{3 K} y_{M}-\left(\frac{2^{3 K}-1}{7}\right)>2^{3 K}\left(\frac{2^{3 K+2}+3}{7 \cdot 2^{3 K+2}}\right)-\left(\frac{2^{3 K}-1}{7}\right)=\frac{1}{4} .
$$

By statement (2) of Lemma 2.4, the solution $\left\{\left(x_{n}, y_{n}\right)\right\}_{n=M+5 K+5}^{\infty}$ is $P_{5}^{1}$.

We now give the proof of Theorem 2.1 when $\left(x_{M}, y_{M}\right)$ is in $l_{4}=\{(x, y): x=0, y<0\}$.

Lemma 2.9. Suppose there exists an integer $M \geq 0$ such that $\left(x_{M}, y_{M}\right) \in l_{4}$. Then the following statements are true.

(1) If $-9 / 7<y_{M}<0$, then $\left\{\left(x_{n}, y_{n}\right)\right\}_{n=M}^{\infty}$ is eventually the equilibrium solution.

(2) If $y_{M}=-9 / 7$, then the solution $\left\{\left(x_{n}, y_{n}\right)\right\}_{n=M+1}^{\infty}$ is $P_{5}^{2}$.

(3) If $y_{M}<-9 / 7$, then the solution $\left\{\left(x_{n}, y_{n}\right)\right\}_{n=M}^{\infty}$ is eventually $P_{5}^{1}$.

Proof. (1) We will first show that statement (1) is true. So suppose $-9 / 7<y_{M}<0$.

Case 1. Suppose $-1 \leq y_{M}<0$. Then

$$
\begin{gathered}
x_{M+1}=\left|x_{M}\right|-y_{M}-1=-y_{M}-1 \leq 0, \\
y_{M+1}=x_{M}-\left|y_{M}\right|=y_{M} .
\end{gathered}
$$

In particular, $-1<x_{M+1} \leq 0$ and $y_{M+1}=-x_{M+1}-1$, and so by Lemma 2.2, $\left\{\left(x_{n}, y_{n}\right)\right\}_{n=M+2}^{\infty}$ is the equilibrium solution.

Case 2. Suppose $-5 / 4 \leq y_{M}<-1$. By statements (1) and (2) of Lemma 2.5, $x_{M+4}=0$ and $y_{M+4}=-4 y_{M}-5$. Then

$$
\begin{gathered}
x_{M+5}=\left|x_{M+4}\right|-y_{M+4}-1=4 y_{M}+4<0, \\
y_{M+5}=x_{M+4}-\left|y_{M+4}\right|=-4 y_{M}-5 .
\end{gathered}
$$

Thus $-1 \leq x_{M+5}<0$ and $y_{M+5}=-x_{M+5}-1$, and so by Lemma 2.2, $\left\{\left(x_{n}, y_{n}\right)\right\}_{n=M+6}^{\infty}$ is the equilibrium solution.

Case 3. Suppose $-9 / 7<y_{M}<-5 / 4$. By statements (1) and (2) of Lemma 2.5, $x_{M+4}=0$ and $y_{M+4}=-4 y_{M}-5$. Note that $0<y_{M+4}<1 / 7$ and so by statement (1) of Lemma 2.8, $\left\{\left(x_{n}, y_{n}\right)\right\}_{n=M+4}^{\infty}$ is eventually equilibrium solution. 
(2) We will next show that statement (2) is true. Suppose $y_{M}=-9 / 7$. By direct calculations we have $\left(x_{M+1}, y_{M+1}\right)=(2 / 7,-9 / 7)$. So the solution $\left\{\left(x_{n}, y_{n}\right)\right\}_{n=M+1}^{\infty}$ is $P_{5}^{2}$.

(3) Finally, we will show that statement (3) is true. Suppose $x_{M}=0$ and $y_{M}<-9 / 7$.

Case 1. Suppose $-3 / 2<y_{M}<-9 / 7$. By statements (1) and (2) of Lemma 2.5, we have $x_{M+4}=$ 0 and $y_{M+4}=-4 y_{M}-5$. Note that $1 / 7<y_{M+4}<1$ and so by statement (3) of Lemma 2.8, the solution $\left\{\left(x_{n}, y_{n}\right)\right\}_{n=M+4}^{\infty}$ is eventually $P_{5}^{1}$.

Case 2. Suppose $y_{M} \leq-3 / 2$. By statement (3) of Lemma 2.5, the solution $\left\{\left(x_{n}, y_{n}\right)\right\}_{n=M+4}^{\infty}$ is $P_{5}^{1}$.

We now give the proof of Theorem 2.1 when $\left(x_{M}, y_{M}\right)$ is in $l_{1}=\{(x, y): x \geq 0, y=0\}$.

Lemma 2.10. Suppose there exists an integer $M \geq 0$ such that $\left(x_{M}, y_{M}\right) \in l_{1}$. Then the following statements are true.

(1) If $0 \leq x_{M}<1 / 7$, then $\left\{\left(x_{n}, y_{n}\right)\right\}_{n=M}^{\infty}$ is eventually the equilibrium solution.

(2) If $x_{M}=1 / 7$, then the solution $\left\{\left(x_{n}, y_{n}\right)\right\}_{n=M+3}^{\infty}$ is $P_{5}^{2}$.

(3) If $x_{M}>1 / 7$, then the solution $\left\{\left(x_{n}, y_{n}\right)\right\}_{n=M}^{\infty}$ is eventually $P_{5}^{1}$.

Proof. (1) We will first show that statement (1) is true. So suppose $0 \leq x_{M}<1 / 7$ and $y_{M}=0$. By statement (3) of Lemma 2.6, $x_{M+6}=0$ and $y_{M+6}=8 x_{M}-1$. In particular, $-1<y_{M+6}<1 / 7$ and so by statement (1) of Lemma 2.8 and statement (1) of Lemma 2.9, $\left\{\left(x_{n}, y_{n}\right)\right\}_{n=M+6}^{\infty}$ is eventually the equilibrium solution.

(2) We will next show that statement (2) is true. Suppose $x_{M}=1 / 7$. By direct calculations we have $\left(x_{M+3}, y_{M+3}\right)=(2 / 7,-9 / 7)$. Thus the solution $\left\{\left(x_{n}, y_{n}\right)\right\}_{n=M+3}^{\infty}$ is $P_{5}^{2}$.

(3) Finally, we will show statement (3) is true.

First consider the case $1 / 7<x_{M} \leq 1 / 4$. By statement (3) of Lemma 2.6, $x_{M+6}=0$ and $y_{M+6}=8 x_{M}-1$. Now, $1 / 7<y_{M+6} \leq 1$ and so by statement (3) of Lemma 2.8 , the solution $\left\{\left(x_{n}, y_{n}\right)\right\}_{n=M+6}^{\infty}$ is eventually $P_{5}^{1}$.

Next consider the case $x_{M}>1 / 4$. Then by statements (1) and (2) of Lemma 2.6, if $x_{M} \geq 1$ then $\left\{\left(x_{n}, y_{n}\right)\right\}_{n=M+2}^{\infty}$ is $P_{5}^{1}$, and if $1 / 4<x_{M}<1$ then $\left\{\left(x_{n}, y_{n}\right)\right\}_{n=M+6}^{\infty}$ is $P_{5}^{1}$.

We next give the proof of Theorem 2.1 when $\left(x_{M}, y_{M}\right)$ is in $l_{3}=\{(x, y): x<0, y=0\}$.

Lemma 2.11. Suppose there exists an integer $M \geq 0$ such that $\left(x_{M}, y_{M}\right) \in l_{3}$. Then the following statements are true.

(1) If $-9 / 7<x_{M}<0$, then $\left\{\left(x_{n}, y_{n}\right)\right\}_{n=M}^{\infty}$ is eventually the equilibrium solution.

(2) If $x_{M}=-9 / 7$, then the solution $\left\{\left(x_{n}, y_{n}\right)\right\}_{n=M+1}^{\infty}$ is $P_{5}^{2}$.

(3) If $x_{M}<-9 / 7$, then the solution $\left\{\left(x_{n}, y_{n}\right)\right\}_{n=M}^{\infty}$ is eventually $P_{5}^{1}$.

Proof. (1) We will first prove statement (1) is true. Suppose $-9 / 7<x_{M}<0$.

First consider the case $-1 \leq x_{M}<0$. Then

$$
\begin{gathered}
x_{M+1}=\left|x_{M}\right|-y_{M}-1=-x_{M}-1, \\
y_{M+1}=x_{M}-\left|y_{M}\right|=x_{M} .
\end{gathered}
$$


In particular, $-1<x_{M+1} \leq 0$ and $y_{M+1}=-x_{M}-1$ and so by Lemma 2.2, $\left\{\left(x_{n}, y_{n}\right)\right\}_{n=M+2}^{\infty}$ is the equilibrium solution.

Next consider the case $-9 / 7<x_{M}<-1$. By statements (1) and (2) of Lemma 2.7, $x_{M+4}=0$ and $y_{M+4}=-4 x_{M}-5$. In particular, $-1<y_{M+4}<1 / 7$ and so by statement (1) of Lemma 2.8 and statement (1) of Lemma 2.9, $\left\{\left(x_{n}, y_{n}\right)\right\}_{n=M+4}^{\infty}$ is eventually the equilibrium solution.

(2) We will next show that statement (2) is true. Suppose $x_{M}=-9 / 7$. By direct calculations, we have $\left(x_{M+1}, y_{M+1}\right)=(2 / 7,-9 / 7)$. That is, $\left\{\left(x_{n}, y_{n}\right)\right\}_{n=M+1}^{\infty}$ is $P_{5}^{2}$.

(3) Lastly, we will show that statement (3) is true. Suppose $x_{M}<-9 / 7$.

First consider the case $-3 / 2 \leq x_{M}<-9 / 7$. By statements (1) and (2) of Lemma 2.7, $x_{M+4}=0$ and $y_{M+4}=-4 x_{M}-5$. In particular, $1 / 7<y_{M+4} \leq 1$ and so by statement (3) of Lemma 2.8, the solution $\left\{\left(x_{n}, y_{n}\right)\right\}_{n=M+4}^{\infty}$ is eventually $P_{5}^{1}$.

Next consider the case $x_{M}<-3 / 2$. By statement (3) of Lemma 2.7, the solution $\left\{\left(x_{n}, y_{n}\right)\right\}_{n=M+4}^{\infty}$ is $P_{5}^{1}$.

We next give the proof of Theorem 2.1 when $\left(x_{M}, y_{M}\right)$ is in $Q_{1}=\{(x, y): x>0, y>0\}$.

Lemma 2.12. Suppose there exists an integer $M \geq 0$ such that $\left(x_{M}, y_{M}\right) \in Q_{1}$. Then the following statements are true.

(1) If $y_{M} \leq x_{M}-1$, then the solution $\left\{\left(x_{n}, y_{n}\right)\right\}_{n=M+2}^{\infty}$ is $P_{5}^{1}$.

(2) If $y_{M}>x_{M}-1$, then there exists an integer $N$ such that $\left(x_{M+N}, y_{M+N}\right) \in l_{2} \cup l_{4}$.

Proof. Suppose $x_{M}>0$ and $y_{M}>0$.

Then

$$
\begin{gathered}
x_{M+1}=\left|x_{M}\right|-y_{M}-1=x_{M}-y_{M}-1, \\
y_{M+1}=x_{M}-\left|y_{M}\right|=x_{M}-y_{M} .
\end{gathered}
$$

Case 1. Suppose $y_{M} \leq x_{M}-1$. Then, in particular, $x_{M+1}=x_{M}-y_{M}-1 \geq 0$ and $y_{M+1}=x_{M}-y_{M}>$ 0 . Thus

$$
\begin{gathered}
x_{M+2}=\left|x_{M+1}\right|-y_{M+1}-1=-2, \\
y_{M+2}=x_{M+1}-\left|y_{M+1}\right|=-1,
\end{gathered}
$$

and so statement (1) is true.

Case 2. Suppose $y_{M}>x_{M}-1$. Then, in particular, $x_{M+1}=x_{M}-y_{M}-1<0$.

Subcase 1. Suppose $x_{M}-y_{M}<0$.

Then $y_{M+1}=x_{M}-y_{M}<0$. It follows by a straight forward computation, which will be omitted, that $x_{M+5}=0$. Hence $\left(x_{M+5}, y_{M+5}\right) \in l_{2} \cup l_{4}$.

Subcase 2. Suppose $x_{M}-y_{M} \geq 0$.

Then $y_{M+1}=x_{M}-y_{M} \geq 0$. It follows by a straight forward computation, which will be omitted, that $x_{M+6}=0$. Hence $\left(x_{M+6}, y_{M+6}\right) \in l_{2} \cup l_{4}$, and the proof is complete.

We next give the proof of Theorem 2.1 when $\left(x_{M}, y_{M}\right)$ is in $Q_{3}=\{(x, y): x<0, y<0\}$. 
Lemma 2.13. Suppose there exists an integer $M \geq 0$ such that $\left(x_{M}, y_{M}\right) \in Q_{3}$. Then the following statements are true.

(1) If $y_{M} \geq-x_{M}-1$, then the solution $\left\{\left(x_{n}, y_{n}\right)\right\}_{n=M+2}^{\infty}$ is the equilibrium solution.

(2) If $y_{M}<-x_{M}-1$, then $\left(x_{M+4}, y_{M+4}\right) \in l_{2} \cup l_{4}$.

Proof. By assumption, we have $x_{M}<0$ and $y_{M}<0$.

If $y_{M} \geq-x_{M}-1$, then

$$
\begin{gathered}
x_{M+1}=\left|x_{M}\right|-y_{M}-1=-x_{M}-y_{M}-1 \leq 0, \\
y_{M+1}=x_{M}-\left|y_{M}\right|=x_{M}+y_{M}<0, \\
x_{M+2}=\left|x_{M+1}\right|-y_{M+1}-1=0 \\
y_{M+2}=x_{M+1}-\left|y_{M+1}\right|=-1 .
\end{gathered}
$$

Hence $\left\{\left(x_{n}, y_{n}\right)\right\}_{n=M+2}^{\infty}$ is the equilibrium solution and statement (1) is true.

If $y_{M}<-x_{M}-1$, then it follows by a straight forward computation, which will be omitted, that $x_{M+4}=0$. Thus $\left(x_{M+4}, y_{M+4}\right) \in l_{2} \cup l_{4}$ and statement (2) is true.

We next give the proof of Theorem 2.1 when $\left(x_{M}, y_{M}\right)$ is in $Q_{2}=\{(x, y): x<0, y>0\}$.

Lemma 2.14. Suppose there exists an integer $M \geq 0$ such that $\left(x_{M}, y_{M}\right) \in Q_{2}$. Then the following statements are true.

(1) If $y_{M} \geq-x_{M}-1$, then $\left(x_{M+1}, y_{M+1}\right) \in Q_{3} \cup l_{4}$.

(2) If $y_{M} \leq-x_{M}-3 / 2$, then $\left(x_{M+3}, y_{M+3}\right) \in Q_{1} \cup l_{1}$.

(3) If $y_{M}<-x_{M}-1, y_{M}>-x_{M}-3 / 2$ and $x_{M} \leq-5 / 4$, then $\left(x_{M+4}, y_{M+4}\right) \in Q_{1} \cup l_{1}$.

(4) If $y_{M}<-x_{M}-1, y_{M}>-x_{M}-3 / 2, x_{M}>-5 / 4$ and $y_{M} \leq x_{M}+5 / 4$, then $\left(x_{M+5}, y_{M+5}\right) \in$ $Q_{3} \cup l_{4}$.

(5) If $y_{M}<-x_{M}-1, y_{M}>-x_{M}-3 / 2, x_{M}>-5 / 4$ and $y_{M}>x_{M}+5 / 4$, then $\left(x_{M+6}, y_{M+6}\right) \in$ $Q_{3} \cup l_{4}$.

Proof. Now $x_{M}<0$ and $y_{M}>0$.

(1) If $y_{M} \geq-x_{M}-1$, then

$$
\begin{gathered}
x_{M+1}=-x_{M}-y_{M}-1 \leq 0, \\
y_{M+1}=x_{M}-y_{M}<0 .
\end{gathered}
$$

Thus $\left(x_{M+1}, y_{M+1}\right) \in Q_{3} \cup l_{4}$.

(2) If $y_{M} \leq-x_{M}-3 / 2$, then $x_{M+1}=-x_{M}-y_{M}-1>0$. It follows by a straight forward computation, which will be omitted, that

$$
\begin{aligned}
& x_{M+3}=-2 x_{M}+2 y_{M}-2>0, \\
& y_{M+3}=-2 x_{M}-2 y_{M}-3 \geq 0 .
\end{aligned}
$$

Hence $\left(x_{M+3}, y_{M+3}\right) \in Q_{1} \cup l_{1}$. 
(3) If $y_{M}<-x_{M}-1, y_{M}>-x_{M}-3 / 2$, and $x_{M} \leq-5 / 4$, then $x_{M+1}=-x_{M}-y_{M}-1>0$. It follows by a straight forward computation, which will be omitted, that

$$
\begin{gathered}
x_{M+4}=4 y_{M}>0, \\
y_{M+4}=-4 x_{M}-5 \geq 0 .
\end{gathered}
$$

Thus $\left(x_{4}, y_{4}\right) \in Q_{1} \cup l_{1}$.

(4) If $y_{M}<-x_{M}-1, y_{M}>-x_{M}-3 / 2, x_{M}>-5 / 4$, and $y_{M} \leq x_{M}+5 / 4$, then $x_{M+1}=$ $-x_{M}-y_{M}-1>0$. It follows by a straight forward computation, which will be omitted, that

$$
\begin{array}{r}
x_{M+5}=4 x_{M}+4 y_{M}+4<0 \\
y_{M+5}=-4 x_{M}+4 y_{M}-5 \leq 0
\end{array}
$$

Thus $\left(x_{M+5}, y_{M+5}\right) \in Q_{3} \cup l_{4}$.

(5) Finally, suppose that $y_{M}<-x_{M}-1, y_{M}>-x_{M}-3 / 2, x_{M}>-5 / 4$, and $y_{M}>x_{M}+5 / 4$. Then $x_{M+1}=-x_{M}-y_{M}-1>0$. It follows by a straight forward computation, which will be omitted, that

$$
\begin{array}{r}
x_{M+5}=4 x_{M}+4 y_{M}+4<0 \\
y_{M+5}=-4 x_{M}+4 y_{M}-5>0
\end{array}
$$

Note that

$$
y_{M+5}=-4 x_{M}+4 y_{M}-5>-4 x_{M}-4 y_{M}-5=-x_{M+5}-1
$$

and so by the first statement of this Lemma, $\left(x_{M+6}, y_{M+6}\right) \in Q_{3} \cup l_{4}$.

Thus we see that if there exists an integer $N \geq 0$ such that $\left(x_{N}, y_{N}\right) \notin Q_{4}$, then the proof of Theorem 2.1 is complete. Finally, we consider the case where the initial condition $\left(x_{M}, y_{M}\right) \in Q_{4}=\{(x, y): x>0, y<0\}$.

Lemma 2.15. Suppose there exists an integer $M \geq 0$ such that $\left(x_{M}, y_{M}\right) \in Q_{4}$. Then there exists a positive integer $N \leq 4$ such that $\left(x_{M+N}, y_{M+N}\right) \notin Q_{4}$.

Proof. Without loss of generality, it suffices to consider the case where

$$
\left(x_{M+n}, y_{M+n}\right) \in Q_{4} \quad \text { for } 0 \leq n \leq 3 .
$$

Now $\left(x_{M}, y_{M}\right) \in Q_{4}$, and hence $x_{M}>0$ and $y_{M}<0$.

Thus

$$
\begin{gathered}
x_{M+1}=\left|x_{M}\right|-y_{M}-1=x_{M}-y_{M}-1, \\
y_{M+1}=x_{M}-\left|y_{M}\right|=x_{M}+y_{M} .
\end{gathered}
$$


We have $\left(x_{M+1}, y_{M+1}\right) \in Q_{4}$, and thus

$$
\begin{gathered}
x_{M+2}=\left|x_{M+1}\right|-y_{M+1}-1=-2 y_{M}-2, \\
y_{M+2}=x_{M+1}-\left|y_{M+1}\right|=2 x_{M}-1 .
\end{gathered}
$$

We also have $\left(x_{2}, y_{2}\right) \in Q_{4}$, and hence

$$
\begin{gathered}
x_{M+3}=\left|x_{M+2}\right|-y_{M+2}-1=-2 x_{M}-2 y_{M}-2, \\
y_{M+3}=x_{M+2}-\left|y_{M+2}\right|=2 x_{M}-2 y_{M}-3 .
\end{gathered}
$$

Finally, we have $\left(x_{M+3}, y_{M+3}\right) \in Q_{4}$, and so

$$
\begin{gathered}
x_{M+4}=\left|x_{M+3}\right|-y_{M+3}-1=-4 x_{M}<0, \\
y_{M+4}=x_{M+3}-\left|y_{M+3}\right|=-4 y_{M}-5 .
\end{gathered}
$$

In particular, $x_{M+4}<0$ and hence $\left(x_{M+4}, y_{M+4}\right) \notin Q_{4}$.

\section{Conclusion}

We have presented the complete results concerning the global character of the solutions to System (1.1). We divided the real plane into 8 sections and utilized mathematical induction, proof by iteration, and direct computations to show that every solution of System (1.1) is eventually either the prime period-5 solution $P_{5}^{1}$, the prime period- 5 solution $P_{5}^{2}$, or else the unique equilibrium point $(0,-1)$. The proofs involve careful consideration of the various cases and subcases.

\section{Acknowledgments}

The authors would like to express their gratitude to the Strategic Scholarships Fellowships Frontier Research Networks, the Office of the Commission on Higher Education, and National Center for Genetic Engineering and Biotechnology.

\section{References}

[1] R. L. Devaney, "A piecewise linear model for the zones of instability of an area-preserving map," Physica D, vol. 10, no. 3, pp. 387-393, 1984.

[2] H. O. Peitgen and D. Saupe, Eds., The Science of Fractal Images, Springer, New York, NY, USA, 1991.

[3] E. A. Grove and G. Ladas, Periodicities in Nonlinear Difference Equations, Chapman \& Hall/CRC, Boca Raton, Fla, USA, 2005.

[4] M. R. S. Kulenović and O. Merino, Discrete Dynamical Systems and Difference Equations with Mathematica, Chapman \& Hall/CRC, Boca Raton, Fla, USA, 2002. 